August 9, 2021

\title{
Phase Transitions and Mass Generation in 2+1 Dimensions
}

\author{
G.W.Semenoff ${ }^{a}$, P. Suranyi ${ }^{\mathrm{b}}$, and L. C. R. Wijewardhana ${ }^{\mathrm{b}}$ \\ ${ }^{a}$ Department of Physics, University of British Columbia \\ Vancouver, British Columbia, Canada V6T $1 Z 1$ and \\ ${ }^{b}$ Department of Physics, University of Cincinnati \\ Cincinnati, Ohio, 45221 U.S.A.
}

\begin{abstract}
The possibility that the epsilon expansion can predict the order of phase transitions in three dimensional field theories is examined. For a Hermitean matrix-valued order parameter, the epsilon expansion predicts fluctuation induced first order phase transitions. We analyze two 2+1-dimensional quantum field theories which exhibit spontaneous symmetry breaking and have martix order parameters. Using the large $N$ expansion, we show that these models exhibit second order transitions and discuss the implications for the chiral symmetry breaking transition in 2+1-dimensional QCD for a critical number of quark flavors.
\end{abstract}




\section{Introduction}

In many of the applications of quantum field theory where phase transitions play a role, the order of the transition is often an important question. For example, the generation of nonzero baryon number in the early universe requires, besides interactions violating $B$ and $T$, that the system is out of equilibrium during the process. This happens, most naturally, in conjunction with supercooling and reheating phenomena near a first order phase transition. On the contrary, a second order chiral phase transition is needed in theories of dynamical mass generation, and also in composite Higgs theories, to ensure that the vast array of mass scales generated dynamically lie well below the scale of interactions responsible for the relevant dynamics [目]. The nature of the chiral phase transition in QCD will also affect results of impending heavy ion experiments.

There has been a substantial amount of work on understanding the detailed nature of the chiral symmetry breaking phase transition both in four-dimensional and three-dimensional QCD. Though there are some important differences, the latter, more recently studied case of three-dimensional QCD shares many of the features of its physical, four dimensional relative. The question of dynamical mass generation can be framed in much the same way as in four dimensions and the technical complexity of the problem is very similar. Many of the analytical methods which are used in four dimensions can also be used in three dimensions. This gives a nontrivial test of the methods in a slightly different context. They can furthermore be compared to numerical data which, though still very incomplete (particularly with dynamical fermions), should be easier to obtain in a lower dimension.

Lower dimensional gauge theory can also be of physical interest, as in phenomenological models of certain condensed matter and statistical systems. The existence or non-existence of, and the detailed nature of phase transitions is of great importance for those models.

A variety of methods have been used to investigate the nature of dynamical mass generation in 3 dimensional gauge theories. One of the most important is the solution of SchwingerDyson equations in the quenched ladder approximation. Another very important method is the $\epsilon$-expansion of Wilson and Fisher [2] around $d=4$. (We shall call this the " $4-\epsilon$ expansion.) Other methods include large $N$ expansions, strong coupling expansions, and the $\epsilon$ expansion about two dimensions applied to chiral Lagrangian sigma models (which we shall call the $2+\epsilon$-expansion). 
In this paper we would like to discuss some of the relationships between these methods. Indeed, in the current literature there is an apparent contradiction between the analysis of the $4-\epsilon$-expansion and the use of Schwinger-Dyson equations. We shall comment on some of the issues involved.

\section{Three-dimensional QCD}

Consider the model defined by the action

$$
S=\int d^{3} x\left(\bar{\psi} \gamma \cdot D \psi+\frac{N_{c} N_{f}}{4 e^{2}} \operatorname{tr} F_{\mu \nu}^{2}\right)
$$

with $N_{c}$ colors and $N_{f}$ flavors of quarks: $\psi_{\alpha}^{a}, a=1 \ldots N_{c}, \alpha=1 \ldots N_{f}$. The Dirac matrices are $2 \times 2$ and Hermitean and $D=\partial+i A, F_{\mu \nu}=\partial_{\mu} A_{\nu}-\partial_{\nu} A_{\mu}+i\left[A_{\mu}, A_{\nu}\right]$.

There is no chiral symmetry in 3 dimensions. Nevertheless, mass terms can violate parity and time reversal invariance. Parity transformation is defined as $\psi(x) \rightarrow \sigma_{2} \psi\left(x^{\prime}\right)$, for the fermions, and $A_{\mu}(x) \rightarrow P_{\mu} A_{\mu}\left(x^{\prime}\right)$ for gauge bosons. Here $x_{\mu}^{\prime}=P_{\mu} x_{\mu}$, where $P_{1}=-P_{2}=$ $P_{3}=1$. The mass term, $\psi^{\dagger} \sigma_{3} \psi$ is obviously odd under parity, but preserves $U\left(N_{f}\right)$ global symmetry.

It is known that parity cannot be spontaneously broken in a vector-like gauge theory [16]. For even $N_{f}$ one can have a mass term that is parity invariant, but breaks the flavor symmetry to $S U\left(N_{f} / 2\right) \times S U\left(N_{f} / 2\right) \times U(1)$. Such a mass term is of the form

$$
L_{m}=\bar{\psi} Q_{N_{f}} \psi
$$

where $Q_{N_{f}}$ is a diagonal matrix with a set of $N_{f} / 2$ eigenvalues of 1 and a set of $N_{f} / 2$ eigenvalues of -1 . Then we can define a parity operator which exchanges fermions pairwise between the two sets.

Whether the flavor symmetry is broken is a dynamical question. The symmetry breaking should generate an expectation value of a mass operator and the physical fermions of the resulting model would be massive. This question is complicated by the fact that, as in four dimensional QCD, the model likely confines color charge. It has been speculated that if chiral symmetry is not broken, even this three dimensional theory, in spite of its severe infrared behavior, would not be confining - the screening of the strong long ranged attractive interactions which would be necessary to restore chiral symmetry would also be sufficient to 
relax confinement [3]. As yet, this heuristic picture has little support from rigorous analytical calculations, but is consistent with all known analytic and numerical results.

The question of whether there can be a phase transition between chirally symmetric and non-symmetric phases and if there exists such a phase transition, what is its order, has attracted wide interest. First, we observe that this question is unconventional in the sense that we are not considering the model (1) at finite temperature, as would be the case in

ordinary critical phenomena. Instead, we are studying how the properties of the ground state of (1) change as we vary the relevant parameters, i.e. the so-called quantum critical phenomena. Second, we observe that, in the conventional sense, the action (1) does not contain any coupling constants which can be varied in order to produce this phase transition. The QCD coupling, $e^{2}$, is dimensional and serves to cut off the ultraviolet divergences [4]. Its presence renders the model super-renormalizable. We therefore do not expect any phase transition as we vary $e^{2}$ - it is simply a dimensional parameter which fixes the units by which we measure energy and distance. The only parameters which can be changed are the numbers of colors and flavors, $N_{c}$ and $N_{f}$ respectively. We ask the question, does the ground state of (11) exhibit different phases for different $N_{c}$ and $N_{f}$ ? Furthermore, if we vary these continuously (which of course cannot be done in the physical world since they are integers), what is the order of the phase transition?

\section{Review of investigations of symmetry breaking}

In this Section, we shall review some of the approaches to the dynamical symmetry breaking problem for three dimensional QCD. Generally, they are based on three different limits of the theory:

- the limit where $N_{c}>>N_{f}$ where planar diagrams dominate

- the limit $N_{f}>>N_{c}$ where the bubble diagrams of the large $N_{f}$ expansion dominate

- the conventional lattice strong coupling limit $e^{2} / \Lambda \rightarrow \infty$ where $\Lambda$ is the ultraviolet cutoff

The most thoroughly analyzed limit of this model is that with $N_{f}>>N_{c}$. In that limit, internal gluon exchanges are suppressed by factors of $N_{c} / N_{f}$ and the gauge interactions are 
very similar to those in the Abelian three dimensional electrodynamics. There have been extensive studies of the symmetry breaking problem in the $1 / N_{f}$ expansion using the self consistent Schwinger-Dyson equations [3] [5]. In the leading order, this approximation sums both bubble and rainbow diagrams and obtains [3] [5] the following picture: At large $N_{f}$ the symmetry is unbroken if $N_{f}>N_{f}^{\text {crit }}$, where $N_{f}^{\text {crit }}=\left(64 / 3 \pi^{2}\right)\left(N_{c}^{2}-1\right) / N_{c}$ and is broken if $N_{f}<N_{f}^{\text {crit }}$. The phase transition at $N_{f}^{\text {crit }}$ is of second order. $N_{f}^{\text {crit }}$ is large enough that the large $N_{f}$ approximation is thought to be quite accurate in that region and corrections to $N_{f}^{\text {crit }}$, for example, have been shown to be small in the next-to-leading order.

The limit $N_{c}>>N_{f}$ has seen much less analysis. In this limit, all of the usual planar diagrams of the conventional large $N_{c}$ expansion of QCD contribute to any process. This limit is therefore more likely to exhibit the features of a strongly interacting gauge field theory - chiral symmetry breaking and confinement.

If the latter limit of the theory is indeed chirally non-symmetric and confining, it could be investigated by effective lagrangian methods. The quantum fluctuations in the broken symmetry phase are described by the Grassmanian $S U\left(N_{f}\right) / S U\left(N_{f} / 2\right) \times S U\left(N_{f} / 2\right)$. It can be parameterized as the set of $N_{f} \times N_{f}$ hermitean matrix-valued fields $\phi(x)$ which have $N_{f} / 2$ eigenvalues 1 and $N_{f} / 2$ eigenvalues -1 . Algebraically, this can be expressed as the condition that $\phi^{2}(x)=1$. The effective action is the Grassmanian sigma-model [6]

$$
S_{\text {eff }}=\int d^{3} x \frac{1}{2 F} \operatorname{tr} \partial \phi \cdot \partial \phi+N_{C} 4 \pi \int_{B_{4}} d^{4} x \operatorname{tr} \phi(d \phi)^{4}
$$

where we have added the final, topological term and where $B_{4}$ is a four dimensional space whose boundary is the three dimensional space on which the first term is defined. As has been pointed out by Feretti and Rajeev [6], the presence of the topological term is necessary to break an extra, unwanted symmetry of the first term $\phi(x) \rightarrow-\phi(x)$ which is not a symmetry of QCD. Furthermore, it is also necessary in order to obtain the correct current algebra. We observe that the number of colors, $N_{c}$ can affect this model only through the dependence on the pion coupling constant $F$ on $N_{c}$ and the coefficient of the topological term.

This model is difficult to analyze directly. It is not renormalizable and a large $N_{f}$ expansion corresponds to the as yet intractable summation of planar fat graphs. However, the coupling constant $F$ is naturally of order $N_{f} / N_{c}$, so if the number of colors is much larger than the number of flavors (in order to suppress the planar graphs with many internal 
$\phi$-lines), this model should be described by the weak coupling limit. It has recently been studied in the saddle point approximation [7] with results suggesting the presence of a second order phase transition to the symmetric phase at some critical value of $N_{f} / N_{c}$.

Furthermore, the model without the topological term has been studied in the $2+\epsilon$ expansion by Brezin, Hikami and Zinn-Justin [8]. They showed that it has an ultraviolet stable fixed point at a non-zero value of the coupling constant $F$. In ref. [7] this was interpreted as implying a critical value of $N_{c} / N_{f}$ above which the chiral symmetry is broken and below which the chiral symmetry is restored.

These results are compatible with the general picture of symmetry breaking which we advocate. However, as we shall discuss later, they may not give a complete description of QCD in the present context in that they neglect the presence of massless fermions at the phase transition point. We shall return to this point later.

The phases of three dimensional QCD have also been studied in the strong coupling limit of lattice gauge theories [9]. It was found that the strongly coupled lattice gauge theory is equivalent to a $S U\left(N_{f} / 2\right)$ Heisenberg antiferromagnet with spins taking values in the Lie algebra [10]

$$
H_{\mathrm{AFM}}=\sum_{\text {lattice }} S^{a}(x) S^{a}(y)
$$

where $x$ and $y$ are nearest neighbors on a bipartite lattice, $a=1, \ldots,\left(N_{f} / 2\right)^{2}-1$ and

$$
\left[S^{a}(x), S^{b}(y)\right]=i f^{a b c} S^{c}(x) \delta(x-y)
$$

is the Lie Algebra of $S U\left(N_{f} / 2\right)$. The spins are in the representation given by the rectangular Young tableau with $N_{f} / 4$ rows and $N_{c}$ columns.

Furthermore, it was argued that the Néel order parameter of the antiferromagnet is equivalent to the mass parameter of the lattice gauge theory. Large $N_{c}$ is the classical limit where the ground state has Néel order. The small $N_{c} / N_{f}$ limit is the quantum limit for the antiferromagnet and is though to be disordered, at least in dimensions less than four. However the precise nature of the ground state in that case is not known. Between these limits there is a phase transition which separates the ordered and disordered phases. This transition is believed to occur at a critical line $N_{f} \simeq N_{c}$ and to be of second order. It has been conjectured that this phase transition coincides with the chiral symmetry breaking transition in three dimensional QCD [7]. 
There are numerical studies of $U(1)$ gauge theory with $N_{f}$ flavors [11]. If the phase transition is indeed independent of the color group, these investigations are also relevant. They also provide a critical value for the number of flavors.

\section{Effective bosonic theory and $4-\epsilon$-expansion}

Pisarski and Wilczek [12] and Wilczek [13] used analogies with condensed matter systems and the 4- - -expansion around dimension 4 of Wilson and Fisher [2] to argue that the finite temperature chiral phase transition of massless four dimensional QCD is second order for two flavors and first order for more than two flavors of quarks, irrespective of the color gauge group. The first order transition is fluctuation driven and it changes the simple mean field theory result that one obtains using the Landau-Ginsberg effective action. In the literature this phenomenon is called the Coleman-Weinberg instability [14].

Pisarski has further argued [15] that a similar situation arises in zero temperature three dimensional QCD. In the following we shall review his reasoning.

First of all, it is assumed that the relevant fluctuating degrees of freedom are bilinear combinations of fermion fields averaged over color. They will be denoted by $\chi_{\alpha \beta}(x)$, defined as

$$
\chi_{\alpha \beta}(x)=\sum_{a=1}^{N_{c}}\left\langle\bar{\psi}_{\beta}^{a}(x) \psi_{\alpha}^{a}(x)\right\rangle .
$$

The effective Landau-Ginzburg action for such bilinears in $4-\epsilon$ dimensions is

$$
S_{\mathrm{LG}}=\int d^{4-\epsilon} x\left\{\frac{1}{2} \operatorname{Tr}(\nabla \chi \cdot \nabla \chi)+\frac{8 \pi^{2} \mu^{\epsilon}}{4 !}\left[g_{1}\left(\operatorname{Tr} \chi^{2}\right)^{2}+\frac{g_{2}}{4 !} \operatorname{Tr} \chi^{4}\right]\right\}
$$

Note that the effective theory is independent of $N_{c}$. Though the trace of the right hand side of (6) does not vanish boson $\chi$ in (7) is defined to be traceless. Based on general arguments, the symmetry breaking pattern is such that parity is conserved. [16] That is automatically satisfied for a boson in adjoint representation, but not for the $S U\left(N_{f}\right)$ scalar. Consequently, the $S U\left(N_{f}\right)$ scalar does not participate in the critical dynamics.

Pisarski [15] used $4-\epsilon$ expansion to investigate the phases of the effective theory. The beta functions of the effective theory are

$$
\begin{aligned}
& \beta_{1}=-\epsilon g_{1}+\frac{N_{f}^{2}+7}{3} g_{1}^{2}+2 \frac{2 N_{f}^{2}-3}{3 N_{f}} g_{1} g_{2}+\frac{N_{f}^{2}+3}{N_{f}^{2}} g_{2}^{3} \\
& \beta_{2}=-\epsilon g_{2}+2 g_{1} g_{2}+2 \frac{N_{f}^{2}-9}{3 N_{f}} g_{2}^{2} .
\end{aligned}
$$


There is an ultraviolet stable fixed point at the origin. There are infrared stable fixed points in the perturbative regime only when $N_{f}=2$. (When $N_{f}<\sqrt{5} \sim 2.3$ there is an infrared stable fixed point at $g^{*}=\left(6 \epsilon /\left(N_{f}^{2}+7\right), 0\right)$ where the model (ఫ) is in the same universality class as the $O\left(N_{f}^{2}-1\right)$ non-linear sigma model.) When $N_{f} \geq 3$ there are no infrared stable fixed points. The situation is very similar to the one encountered by Coleman and Weinberg [14]. There, the existence of two competing coupling constants allowed the existence of a minimum of the effective potential in the perturbative regime, leading to a fluctuation induced first order phase transition. Conditions for the existence of first order phase transitions can be obtained from the $\beta$-functions alone as it has been shown a long time ago [17]. The condition, beside the absence of infrared stable fixed points, is that the renormalization group trajectories cross the stability line,

$$
4\left(g_{1}+g_{2}\right)+\beta_{1}\left(g_{1}, g_{2}\right)+\beta_{2}\left(g_{1}, g_{2}\right)=0
$$

a line at which the potential is minimized. Provided the potential vanishes at zero field, this should happen in the region where the potential is negative, $g_{1}+g_{2}<0$. For $N \geq 3$ the trajectories generated by the beta functions of (8) do cross the line of stability. The conclusion of Pisarski is that there is a first order phase transition for $N_{f} \geq 3$, and parity conserving mass is generated spontaneously for all $N_{f}$.

This result seems incompatible with the picture of a chiral phase transition at some critical value of $N_{c} \sim N_{f}$ which we have described in the previous sections. In the remainder of this Section we shall list some of the possible reasons for this apparent lack of agreement.

- In the conventional applications of the $4-\epsilon$-expansion to study the critical behavior of a finite temperature 4 dimensional theory, the fermions cannot contribute to critical fluctuations because they have antisymmetric boundary conditions in the time direction and thus their 'mass' is $O(1 / \beta)$. Therefore, fermionic degrees of freedom need not be taken into account in the computation of the $\beta$-function. In the zero temperature 3 dimensional theories which we are studying here, however, the mass of fermions vanishes at the chiral transition point, thus their contribution is important and should also be taken into account. Thus if the theory has only four fermi interactions and no gauge interactions it belongs to the universality class of Yukawa theories. Therefore to do a reliable $\epsilon$ expansion analysis one should add an appropriate Yukawa coupling to the 
scalar theory in equation (7) and look for fixed points of the resulting beta functions. In the case of QCD3 the situation is somewhat complicated. If confinement effects are small, one must also include gauge interactions in the computation of the beta functions. It has been conjectured that when the number of fermion flavors exceeds a critical value, the long range attractive gauge interactions get screened leading not only to chiral symmetry restoration but also to deconfinement citeappelquist2. If this is the case Pisarski's calculation should be appropriately modified to take into account the added complications. A calculation of effective scalar potential for QCD3 including gauge and fermion contributions has been carried out in the large flavor limit [18. It is seen that the effective potential shows non analytic behavior as the critical point is approached from the symmetric phase.

- The symmetries of the effective action (7) are not quite the same as that of three dimensional QCD described by (面). Action (7) has a reflection symmetry, $\chi \rightarrow-\chi$, which is distinct from parity and which is absent from the original gauge theory. This symmetry cannot be broken by relevant terms in the framework of the Landau-Ginzburg approach. It is broken by the topological term in the sigma model (3). However, it is impossible to take into account the influence of the topological term in the epsilon expansion since it cannot be continued in dimensions.

- There are known examples of non-perturbative infrared stable fixed points. Even in the scenario of the $4-\epsilon$-expansion starting with a Landau-Ginzburg effective action, that is valid in the large $N_{c}$ limit, it is very possible that there exists a non-perturbative infrared stable fixed point. Such fixed points are known to occur in 3-dimensional models with fermions and four-fermion interactions [2, 19]. For example, consider [20]

$$
S=\int d^{3} x\left(\bar{\psi}(\gamma \cdot \partial+\phi) \psi+\frac{N \Lambda}{2 \lambda} \phi^{2}\right)
$$

Eliminating $\phi$ by using its equation of motion, produces the 4-fermi coupling $-\frac{\lambda}{2 N}(\bar{\psi} \psi)^{2}$, which is invariant under a global $S U(N)$ flavor symmetry. This model is renormalizable in the large $N$ expansion. Integrating the Fermions gives the effective scalar field theory

$$
S_{\text {eff }}=-N \operatorname{Tr} \ln (\gamma \cdot \partial+\phi)+\frac{N \Lambda}{2 \lambda} \int \phi^{2}
$$


for which $1 / N$ is the coupling constant. It is known that this model has a nonperturbative fixed point at large $\lambda$. Note the difference between this model and gauge theory. Since the former is strictly renormalizable, the ultraviolet cut-off $\Lambda$ appears as a dimensionful parameter in addition to the dimensionful coupling $\lambda$, thus providing an additional parameter $\lambda \Lambda$. It is this parameter which is tuned to obtain critical behavior.

- The 4- $\epsilon$ expansion is known not to be reliable in some models. The Higgs-gauge model with $N$ complex scalars, which is a model for superconductors, was found by Halperin, Lubensky, and Ma [21] to have a first order phase transition, due to the lack of infrared stable fixed points. This is a generalization of the Coleman-Weinberg argument. It is known that this system in the Type II regime (roughly speaking the Higgs mass is larger than the gauge mass) has the same critical properties as the smectic-nematic phase transition in liquid crystals. Experimentally, the latter system was found to have a second order transition with $X Y$ exponents. The problem has been studied by Monte Carlo simulations [22]. It was shown that at $\epsilon=1$ there is a new fixed point with $X Y$ exponents, which is absent at $\epsilon=0$. March-Russell [23] came to the same conclusion by studying the gauge interactions of $N$ complex $p$-vector Higgs bosons in $U(p)$-gauge theory and comparing $4-\epsilon$ and $2+\epsilon$ expansions. In general, sigma models have been studied in the $2+\epsilon$ expansion by Brezin, Hikami, and Zinn-Justin. They found fixed points and nonzero coupling for $\epsilon>0$. This is in contradiction with results obtained when one investigates linear sigma models in $4-\epsilon$ expansion, though the physical degrees of freedom driving the phase transition and the universality class are believed to coincide in the two models.

If, as we have noted above is possible, the analysis of the $4-\epsilon$ expansion is reliable in the limit of large $N_{c}$, it is still implies that the chiral phase transition is a fluctuation induced first order transition. This comes about by the following reasoning: In the zero temperature gauge theory defined by action (1), the parameters are $N_{f}$, and $N_{c}$.

The coefficients of the operators in the effective Landau-Ginzburg theory (7) may depend on the parameters of the model. In the case of QCD in $2+1$ dimensions, the only relevant parameters are $N_{c}$ and $N_{f}$. There is a phase transition if it of possible to tune $N_{c}$ and $N_{f}$ so that the quadratic terms in the Landau-Ginsberg potential vanish. This would give a critical 
curve in the $N_{c}-N_{f}$-plane. The results of [5] imply that this curve is approximately linear, $N_{f} \simeq N_{c}$. The question of whether the transition is second order or a fluctuation induced first order transition remains. It is not clear that the analyses of the phase transition described in Section 3 distinguish a second order transition from one which is weakly first order. This could in principle be resolved using an improved $4-\epsilon$ expansion. Also, the model which we construct in the next Section has the same flavor symmetries as 2+1-dimensional QCD and has a second order transition. If they indeed lie in the same universality class, this would indicate that the chiral transition at $N_{f} \sim N_{c}$ in QCD is indeed second order.

\section{$5 \quad$ A gauge model with global symmetry $U\left(N_{f}\right) \otimes Z_{2}^{\text {parity }}$}

In this Section we shall construct a model which has the same global symmetries as QCD, is renormalizable in the large $N$ expansion, and has a second order chiral phase transition. Since the model shares the Landau-Ginzburg potential with QCD, claims of a proof of first order phase transition based on the Landau-Ginzburg potential and the $4-\epsilon$-expansion are invalidated. The model will consist of four-fermion interactions which drive the phase transition. To illustrate, we begin with a simpler version, a theory with only four fermion interactions and where the color symmetry is not gauged.

\subsection{A toy model with 4-Fermion interaction}

Let us begin this section with a toy model with 4-fermion interactions with $N_{f}$ "flavors" and $N_{c}$ "colors" of fermions $\psi_{a}^{\alpha} a=1 \ldots N_{c}, \alpha=1 \ldots N_{f}$ and an action which is a generalization of (9)

$$
S=\int d^{3} x\left(\bar{\psi}(\gamma \cdot \partial+\phi) \psi+\frac{N_{c} \Lambda}{2 \lambda} \operatorname{tr}\left(\phi^{2}\right)\right) .
$$

Here $\phi_{\alpha \beta}$ is a traceless $N_{f} \times N_{f}$ Hermitian matrix. Integrating over $\phi$ gives the four-fermion interaction

$$
-\frac{\lambda}{2 N_{c} \Lambda} \int d^{3} x \bar{\psi}_{a} T_{A} \psi_{a} \bar{\psi}_{b} T_{A} \psi_{b}
$$

where both flavor and color indices have been omitted. $T_{A}$ is the generator of the flavor symmetry group, $S U\left(N_{f}\right)$. One could modify interaction term (12) by including an isoscalar four-fermi interaction, $(\bar{\psi} \psi)^{2}$. That case will be discussed briefly later.

This model can be readily analyzed in the large $N_{c}$ expansion. If the color group were gauged, the necessity of summing planar diagrams would render this expansion intractable. 
In the following section we shall suggest a way to overcome this difficulty. In this section we shall study the model (11).

The fermion fields can be integrated out to give in leading order of $N_{c}$ the following effective potential for the scalar field

$$
V[\bar{\phi}]=N_{c} \mathbf{t r}\left[\left(\frac{\Lambda}{\lambda}-\frac{\Lambda}{\pi^{2}}\right) \frac{\bar{\phi}^{2}}{2}+\frac{1}{6 \pi}\left[\bar{\phi}^{2}\right]^{3 / 2}\right]
$$

where the trace is over "flavor" indices. This potential predicts a second order phase transition at the point where the effective mass parameter

$$
t \equiv \frac{\Lambda}{\lambda}-\frac{\Lambda}{\pi^{2}}
$$

vanishes. When $t<0$, the field $\phi$ acquires a vacuum expectation value, breaking the $U\left(N_{f}\right)$ symmetry. The tree approximation gives the mean field value, 0 , for the exponent $\gamma$, defined by

$$
V^{\mathrm{crit}}[\bar{\phi}] \sim \operatorname{tr}|\bar{\phi}|^{d(1+\gamma)}
$$

As we can see there is still no dependence of the universality class on $N_{c}$ at tree level.

The effect of higher order corrections will be sketched below. In $O\left(1 / N_{c}\right)$ one has to calculate contributions coming from the fluctuations of the fields. The corrected potential has the form

$$
V[\bar{\phi}]=N_{c}\left[\frac{t}{2} \operatorname{tr} \bar{\phi}^{2}+\frac{1}{6 \pi} \operatorname{tr}\left(\bar{\phi}^{2}\right)^{3 / 2}+\frac{1}{N_{c}} \mathbf{T R} \ln \Delta\right],
$$

where $\mathbf{T R}$ is a trace over spacetime as well as flavor indices and

$$
\Delta_{i j k l}(x, y)=\frac{1}{\lambda}\left(\delta_{i l} \delta_{j k}-\frac{1}{N_{f}} \delta_{i j} \delta k l\right) \delta(x-y)+\operatorname{Tr}\left(x\left|\frac{1}{\gamma \cdot \partial+\bar{\phi}}\right| y\right)_{i l}\left(y\left|\frac{1}{\gamma \cdot \partial+\bar{\phi}}\right| x\right)_{j k},
$$

is the self-energy of the scalar to order $N_{c}^{-1}$.

The global flavor symmetry can be used to diagonalize the order parameter $\bar{\phi}$. Here, we shall assume the symmetry breaking pattern (when $N_{f}$ is even) which preserves parity and an $U\left(N_{f} / 2\right) \times U\left(N_{f} / 2\right)$ flavor symmetry

$$
\bar{\phi}=\operatorname{diagonal}(m, m, \ldots, m,-m,-m, \ldots,-m) \text {. }
$$

Then, the effective potential has the form[24]

$$
V_{\mathrm{eff}}=\frac{N_{c} N_{f}}{2} t m^{2}+\frac{N_{c} N_{f}}{6 \pi} m^{3}+\frac{2 N_{f}^{2}}{3 \pi^{3}} m^{3} \ln \frac{m}{\mu} .
$$


The cubic term of the effective potential comes from the fermion ring vacuum diagram, while the last term from the boson ring vacuum diagram. The effective mass parameter $t$ also renormalizes at this order. We have also cancelled a logarithmic ultraviolet divergence by a wave-function renormalization for the scalar field, $m \rightarrow z m$ and appropriate choice of $z$.

The logarithmic term in the effective potential is reminiscent of a similar term in the Coleman-Weinberg [14] effective potential in four dimensional theories. Furthermore, as in the Coleman-Weinberg analysis, the critical model (at $t=0$ ) indeed has a minimum away from the origin and an apparent first order phase transition with the exponentially small order parameter

$$
m_{0}=\mu \exp \left(-\frac{\pi^{2}}{4} \frac{N_{c}}{N_{f}}\right)
$$

However, as in the Coleman-Weinberg scenario with one coupling constant, this minimum is at a point outside of the perturbative regime - it is for an exponentially small order parameter and the logarithm in the effective potential invalidates the perturbative result in the region where the order parameter is close to zero.

As usual, the trouble with the effective potential near $m=0$ can be fixed using the renormalization group. This amounts to understanding how higher orders in logarithms would contribute to the effective potential. For the present case (unlike in the four-dimensional model considered by Coleman and Weinberg) we shall argue in the Appendix that the corrections exponentiate to produce an effective potential of the form

$$
V_{\text {eff }}=\frac{N_{c} N_{f}}{2} t m^{2}+\frac{N_{c} N_{f}}{6 \pi} m^{3\left[1+4 N_{f} /\left(3 \pi^{2} N_{c}\right)\right]} .
$$

This improved effective potential exhibits a second order phase transition at $t=0$.

The exponent $\gamma$ defined in (15) is $O\left(1 / N_{c}\right), \gamma=4 N_{f} /\left(3 \pi^{2} N_{c}\right)$. The exponent is identical with the anomalous dimension of the boson. In other words, the universality class depends on $N_{c}$. In the broken phase, $\mu^{2}<0$, the eigenvalues of matrix $\langle\phi\rangle$ are $m_{i}= \pm m_{0}$, where $m_{0} \sim(|t|)^{1 /(1+3 \gamma)}$.

It is worth mentioning what happens if a flavor neutral term is included in the fourfermion interaction. Then in addition to the adjoint representation scalar boson (isotensor boson), one is forced to introduce a flavor neutral (isoscalar) boson as well. The isotensor and isoscalar bosons have, in general, different couplings to fermion pairs. On one hand, 
new divergent diagrams appear and both couplings start to run. The anomalous dimension of bosons is not any more twice the anomalous dimension of fermions. On the other hand, some crucial features of the pure isotensor theory survive. The logarithmic derivatives of couplings and the anomalous dimensions are independent of the couplings constants; they depend on $N_{f}$ and $N_{c}$ only. The running fermion-boson coupling constant exponentiate into forms $g \sim m^{\lambda\left(N_{f}\right) / N_{c}}$. In a similar manner, the three boson couplings exponentiate as well. Thus, even in this case, the effective potential exponentiates into a pure $N_{f}$ and $N_{c}$ dependent power of the the expectation value of the field, $m$. The phase transition is of second order and critical exponents are dependent on $N_{f}$ and on $N_{c}$. Details of the discussion of this model will be given elsewhere.

\subsection{Gauging the "color" group: Chern-Simons gluo-dynamics}

The four-fermi theory which we have been discussing has a second order phase transition. However, the flavor symmetries is very different from that of $2+1$-dimensional QCD. One can come closer to QCD and remove the color degeneracy of the model by gauging the color symmetry. We shall introduce gauge fields in such a way as to preserve, as much as possible, the solvability of the four-fermion model in the large $N_{c}$ limit.

Consider the action

$$
\begin{aligned}
S= & \int d^{3} x\left(\bar{\psi}\left(\gamma \cdot D_{A}+\phi+M\right) \psi+\bar{\chi}\left(\gamma \cdot D_{B}+\phi-M\right) \chi\right. \\
& \left.+\frac{N_{c} \Lambda}{2 \lambda} \operatorname{tr}\left(\phi^{2}\right)+\frac{N_{c}}{2 g^{2}} \operatorname{tr}\left(A d A+\frac{2}{3} A^{3}-B d B-\frac{2}{3} B^{3}\right)\right)
\end{aligned}
$$

where $D_{A}=\partial+i A$ and $D_{B}=\partial+i B$. Here, we have assumed that there are an even number of colors and have gauged the group $S U\left(N_{c} / 2\right) \times S U\left(N_{c} / 2\right)$. As a kinetic term for the gauge field, we have introduced the Chern-Simons terms with a dimensionless coupling constant

$g$. This makes the gluon non-propagating. It is very important to observe that even though there are Chern-Simons terms in the theory, it is still parity invariant. In fact, this theory has the same flavor symmetries as QCD: it is parity symmetric, the parity transformation, besides the obvious spacetime transformation, also interchanges the two types of fermions $\chi$ and $\psi$ and the two types of gauge fields $A$ and $B$. This symmetry insures that if there is no bare fermion mass it will not be generated to any finite order in perturbation theory.

This theory also has the flavor symmetry $U\left(N_{f}\right)$ and one can study the possibility of a 
phase transition with symmetry breaking pattern $U\left(N_{f}\right) \rightarrow U\left(N_{f} / 2\right) \times U\left(N_{f} / 2\right)$. Its one large difference with QCD is that here the fermions have a bare mass, $M$. They are not massless at the critical point unless we also tune $M=0$. This model thus allows us to explore two possibilities, one which is like QCD with $M=0$ and where massless fermions are important to the critical behavior and the other where $M \neq 0$, fermions are all massive in the region of $\phi \sim 0$ and one would expect that perhaps the Landau-Ginsburg theory and epsilon expansion gave an accurate description of the fluctuating degrees of freedom.

For the gauge interactions, to obtain the large $N_{c}$ limit one must sum all of the planar diagrams which contribute to the effective potential. The leading order is $N_{c}^{2}$ and contains no fermion loops. It therefore does not couple to the order parameter $\bar{\phi}$ and does not contribute to the effective potential for $\bar{\phi}$. The next to leading order, $\sim N_{c}$, contains one fermion loop, no scalar lines and all insertions of planar gluon lines. The problem of summing all contributions of planar gluons is as yet intractable. Here, we shall remedy this situation by using a simultaneous large $N_{c}$ and small $g^{2}$ expansion [25]. In this expansion, unlike conventional gluo-dynamics where the gluons would have a Yang-Mills action, the ChernSimons gluo-dynamics is infrared finite, i.e. infrared divergences do not ruin the perturbative expansion [26].

Then, when $M=0$ (the QCD case), the order $g^{2} N_{c}^{0}$ contribution to the effective potential vanishes. Then, in this model, the effective potential is given by (19) with corrections being of order $N_{c}^{k} g^{2 p}$ with $k+p \geq 1$. Furthermore, since the gauge interactions do not contribute to the logarithmic divergence to the order we have considered, the renormalization group analysis as well as the result (21) are also valid. Thus, this theory has a second order phase transition and has the same flavor symmetry as QCD.

The analysis of this problem when $M \neq 0$ is more difficult and thus far is done only to the leading order. There, the effective potential has the form

$$
V_{\text {eff }}^{M}[\bar{\phi}]=N_{c} \operatorname{tr}\left\{\begin{array}{cc}
\frac{t}{2} M^{2}+\frac{M^{3}}{6 \pi}+\left(\frac{t}{2}+\frac{M}{2 \pi}\right) \bar{\phi}^{2} & |\bar{\phi}| \leq M \\
\frac{t}{2} M^{2}+\frac{M^{2}}{2 \pi}|\bar{\phi}|+\frac{1}{6 \pi}|\bar{\phi}|^{3} & |\phi| \geq M
\end{array}\right\}
$$

This system has a marginally first order behavior. When $t>t_{c}=-M / 2 \pi$ the minimum is at $\bar{\phi}=0$. Then at $t=t_{c}$ the effective potential has zero curvature in the region $|\bar{\phi}| \leq M$ and is convex where $|\bar{\phi}|>M$. When $t>t_{c}$ the minimum begins at $|\bar{\phi}|=M$ and increases as $t$ decreases. This is very similar to a first order behavior: the order parameter $\bar{\phi}$ jumps from $\bar{\phi}=0$ to $|\bar{\phi}|=M$ at $t=t_{c}$. 
It is intriguing that this "almost first order" behavior occurring for this case is probably closer to the prediction of the epsilon expansion than the case of pure QCD. However, even here we conjecture that higher order corrections smooth the behavior of the effective potential and the phase transition remains second order. Detailed behavior of this model in the next order of expansion are interesting and are the subject of further study.

\section{Conclusion}

In this Paper, we have analyzed a pure four-fermion model and a gauged four-fermion model in the large $N_{C}$ limit. We have shown that both of these models exhibit second order phase transitions.

Perturbatively, the models appear to have fluctuation induced first order transitions. However, we have shown that higher order corrections lead to exponentiation of logarithmic terms and restoration of second order behavior.

In the gauged four-fermi model, we could consider two cases - one where the fermions are massless at the phase transition and the other where they are massive. In the case where they are massless, at least to the order of perturbation theory which we considered, the nature of the phase transition was identical to that in the pure four-fermion theory. In the case where the fermions are massive, however, the behavior was markedly different - it exhibited an "almost first order" phase transition. It is intriguing that the $4-\epsilon$ expansion predicts a fluctuation induced first order transition for this model. It is also interesting that as the fermion bare mass goes to zero, the transition becomes second order (the jump in the order paramater as the coupling is varied through the critical point is proportional to the bare mass).

\section{A Appendix}

The exponentiation of the potential is proven by the method of renormalization group. The field theory we consider is defined by action (11). The fermion bubble corrections are summed to give a modified boson propagator, which has the form

$$
D_{i j}^{-1}(p)=\frac{1}{2}\left(\frac{\Lambda}{\lambda}-\frac{\Lambda}{\pi^{2}}\right)+\frac{m}{2 \pi}+\frac{p^{2}+\left(m_{i}+m_{j}\right)^{2}}{4 \pi p} \tan ^{-1} \frac{p}{2 m}
$$




$$
=\frac{1}{2}\left(\frac{\Lambda}{\lambda}-\frac{\Lambda}{\pi^{2}}\right)+\frac{p}{8}+\frac{\left(m_{i}+m_{j}\right)^{2}}{8 p}-\frac{m\left[3\left(m_{i}+m_{j}\right)^{2}-4 m^{2}\right]}{6 \pi p^{2}}+O\left(m^{4} p^{-3}\right),
$$

where $m_{i}= \pm m$ is the mass of the $i$ th fermion.

The renormalization group functions can easily be calculated in $O\left(N_{f} / N_{c}\right)$. The only primitive divergent diagram is the fermion self-energy correction diagram in this order. Thus, the coupling constant does not run. The logarithmically divergent part of the fermion self energy diagram is

$$
\Delta S_{F}^{-1}(k) \simeq \frac{4 N_{f}}{3 N_{c} \pi^{2}} \not k \ln \frac{\Lambda}{\mu} .
$$

The boson propagator gets logarithmic corrections only due to the dissociation of the boson into two fermions. Thus, the leading order anomalous dimension of the boson is twice the anomalous dimension of the fermion. In other words, as shown by (25),

$$
\gamma_{b}=\frac{4 N_{f}}{3 N_{c} \pi^{2}}
$$

At the critical point, the Callan-Symanzik equation

$$
\left(\frac{\partial}{\partial t}+\gamma_{b} m \frac{\partial}{\partial m}\right) V(m)=0
$$

has the boundary condition

$$
\lim _{N_{f} / N_{c} \rightarrow 0} \frac{V_{0}}{N_{c} N_{f}}=\frac{1}{6 \pi} m^{3} .
$$

Here $t=\log \mu / m$. Then the solution of the Callan-Symanzik equation is straightforward.

$$
V_{\text {eff }}=\frac{1}{6 \pi} m^{3} \exp \left\{-3 \int_{0}^{t} \mathrm{~d} t^{\prime} \frac{\gamma_{b}\left(t^{\prime}\right)}{1-\gamma_{b}\left(t^{\prime}\right)}\right\} .
$$

Substituting (26) into (29) gives exponentiated form (21).

\section{ACKNOWLEDGEMENTS}

The work of G. S. is supported by the Natural Science and Engineering Research Council of Canada. The work of P. S. and of L. C. R. W. is supported in part by the United States Department of Energy under grant no. DE-FG02-84ER40153. L.C.R.W. thanks T. Appelquist and J. Terning for valuable discussions. 


\section{References}

[1] Y. Nambu, in the Proceedings of the XI International Symposium on Elementary Particle Physics, Kazimierz, Poland, 1988, edited by Z. Adjuk, S. Pokorski, and A. Trautmann (World Scientific, Singapore, 1989);

T. Appelquist, T. Takeuchi, M. Einhorn, and L.C.R. Wijewardhana, Phys. Lett. B220, 223 (1989);

V.A. Miransky, M. Tanabashi, and K. Yamawaki, Phys. Lett. B221, 1043 (1989); ibid. Mod. Phys. Lett. A4, 1043 (1989);

R.S. Chivukula, A.G. Cohen, and K. Lane, Nucl. Phys. B343, 554 (1990);

R.S. Chivukula, M. Golden, and E.H. Simmons, Phys. Rev. Lett. 70, 1587 (1993);

W.A. Bardeen, C.T. Hill, and D-U. Jungnickel, Preprint, FERMI-PUB-93/035-T;

[2] K.Wilson, Phys.Rev. D14, 2911 (1974).

[3] T. Appelquist and D. Nash, Phys. Rev. Lett. 64, 721 (1990);

[4] This is somewhat analogous to four dimensional QCD where the dimensional transmutation which accompanies asymptotic freedom changes the coupling constant into the mass scale. Both theories are weakly coupled at high energies.

[5] T. Appelquist, D. Nash and L. C. R. Wijewardhana, Phys. Rev. Lett. 60, 2575 (1988); D. Nash, Phys. Rev. Lett. 62, 3024 (1989);

[6] G. Ferretti and S. G. Rajeev, Phys. Rev. Lett. 69, 2033 (1992); G. Ferretti, S.G. Rajeev, Z. Yang, Int.J.Mod.Phys. A7, 7989 (1992); G. Ferretti, S.G. Rajeev, Z. Yang, Int.J.Mod.Phys. A7, 8001 (1992);

[7] M.-C. Diamantini, G. W. Semenoff and P. Sodano, Phys. Rev. Lett. 70, 3438 (1993);

[8] E.Brezin, S.Hikami and J.Zinn-Justin, Nucl.Phys.B165, 128(1980).

[9] G.Semenoff, Mod.Phys.Lett. A17, 2811 (1992);

E.Langmann and G.Semenoff, Phys. Lett. B297, 175 (1992);

M.C.Diamantini, P.Sodano, E.Langmann and G.Semenoff, Quantum spin systems and the vacuum structure of strong coupling gauge theory with dynamical fermions, Proc. of Field Theory and Collective Phenomena, Perugia, Italy, June 1992;

M.C.Diamantini, P.Sodano, E.Langmann and G.Semenoff, $S U(N)$ antiferromagnets and the phase structure of QED in the strong coupling limit, Univ. Perugia preprint, 1992.

[10] This is, strictly speaking, only true when $N_{f}$ is a multiple of four. The reduction of the flavor symmetry group from $S U\left(N_{f}\right)$ to $S U\left(N_{f} / 2\right)$ occurs by explicit symmetry breaking by the lattice regularization. In that case, the continuous part of what we refer to as chiral symmetry is actually broken explicitly. However, a discrete chiral symmetry remains and is sufficient to render fermions massless. It is therefore reasonable to ask whether fermion mass is generated in the lattice models. This mass generation is found to occur as Néel order of the effective antiferromagnet.

[11] S. Dagotto, J. Kogut and A. Kocic, Phys. Rev. Lett. 62,1083 (1989); Nucl. Phys. B334, 229 (1990);

[12] R. Pisarski and F. Wilczek, Phys. Rev. D29, 338, 1222, 2423 (1984);

[13] F. Wilczek, 'Remarks on the phase transition in QCD', preprint IASSNS-HEP-92/23; Int.J.Mod.Phys. A7, 3911 (1992); 
[14] S. Coleman and E. Weinberg, Phys. Rev. D7, 1888 (1973);

[15] R. Pisarski, Phys. Rev. D44, 1866 (1991).

[16] C. Wafa and E. Witten, Nucl. Phys. B234, 173 (1984); A.P. Polychronakos, Phys. Rev. Lett. 60, 1920 (1988);

[17] H. Yamagishi, Phys. Rev. D23, 1880 (1981);

[18] T. Appelquist, J. Terning and L. C. R. Wijewardhana, unpublished.

[19] B. Rosenstein, B. Warr, and S. Park, Phys. Lett. B218, 465 (1989); B219, 469 (1989); Phys. Rev. D39, 3088 (1989);

[20] G.W. Semenoff and L.C.R. Wijewardhana, Phys. Rev. D45, 1342 (1992);

[21] B. I. Halperin, T. Lubensky, and S.K. Ma, Phys. Rev. Lett, 32, 292 (1974);

[22] C. Dasgupta and B. I. Halperin, Phys. Rev. Lett. 47, 1556 (1981);

[23] J. March-Russell, On the Possibility of Second-Order Phase transitions in Spontaneously Broken Gauge Theories, Princeton preprint PUPT-92-1328.

[24] The method for computing the integral is outlined in ref. [0].

[25] One could alternatively view this expansion as a large $N_{c}$ expansion where $g^{2}$ is also of order $1 / N_{c}$.

[26] W. Chen, G. Semenoff, and Yong-Shi Wu, Phys. Rev. D44, 1625 (1991). 